\title{
A Mass Balance-Based Semiparametric Approach to Evaluate Neonatal Erythropoiesis
}

\author{
Denison J. Kuruvilla, ${ }^{1}$ John A. Widness, ${ }^{2}$ Demet Nalbant, ${ }^{2}$ Robert L. Schmidt, ${ }^{2}$ \\ Donald M. Mock, ${ }^{3}$ and Peter Veng-Pedersen ${ }^{1,4}$
}

Received 8 July 2015; accepted 25 September 2015; published online 26 October 2015

\begin{abstract}
Postnatal hemoglobin $(\mathrm{Hb})$ production in anemic preterm infants is determined by several factors including the endogenous erythropoietin levels, allogeneic RBC transfusions administered to treat anemia, and developmental age. As a result, their postnatal $\mathrm{Hb}$ production rate can vary considerably. This work introduces a novel $\mathrm{Hb}$ mass balance-based semiparametric approach that utilizes infant blood concentrations of $\mathrm{Hb}$ from the first 30 postnatal days to estimate the amount of $\mathrm{Hb}$ produced and the erythropoiesis rate in newborn infants. The proposed method has the advantage of not relying on specific structural pharmacodynamic model assumptions to describe the $\mathrm{Hb}$ production, but instead utilizes simple mass balance principles and nonparametric regression analysis. The developed method was applied to the $\mathrm{Hb}$ data from 79 critically ill anemic very low birth weight preterm infants to evaluate the dynamic changes in erythropoiesis during the first month of life and to determine the inter-subject variability in $\mathrm{Hb}$ production. The estimated mean $( \pm \mathrm{SD})$ cumulative amount of $\mathrm{Hb}$ produced by the infants over the first month of life was $6.6 \pm 3.4 \mathrm{~g}$ (mean body weight, $0.768 \mathrm{~kg}$ ), and the mean estimated body weight-scaled $\mathrm{Hb}$ production rate over the same period was $0.23 \pm 0.12 \mathrm{~g} / \mathrm{day} / \mathrm{kg}$. A significant positive correlation was observed between infant gestational age and the mean body weight-scaled $\mathrm{Hb}$ production rate over the first month of life $(P<0.05)$. We conclude that the proposed mathematical approach and its implementation provide a flexible framework to evaluate postnatal erythropoiesis in newborn infants.
\end{abstract}

KEY WORDS: anemic preterm infants; neonatal erythropoiesis; pharmacodynamic; postnatal hemoglobin production; very low birth weight.

\section{INTRODUCTION}

All newborn infants experience a decline in blood concentrations of hemoglobin ("Hb levels" hereafter) during the first weeks of life. In healthy term infants, this postnatal drop in Hb levels is well tolerated, does not require therapy, and is commonly referred to as "physiological anemia of infancy" (1-3). In critically ill very low birth weight (VLBW, birth weight $<1500 \mathrm{~g}$ ) and extremely low birth weight (ELBW, birth weight $<1000 \mathrm{~g}$ ) preterm infants, the $\mathrm{Hb}$ levels fall to significantly lower levels than term infants and often require one or more $\mathrm{RBC}$ transfusions as treatment for clinically significant "anemia of prematurity" $(1,3)$.

A major factor responsible for the postnatal decline in $\mathrm{Hb}$ levels is a substantial decline in the rate of erythropoiesis, especially during the first weeks of extra-uterine life (4).

\footnotetext{
${ }^{1}$ Department of Pharmaceutical Sciences and Experimental Therapeutics, College of Pharmacy, University of Iowa, 115 S. Grand Ave. S227, Iowa City, Iowa 52242, USA.

${ }^{2}$ Department of Pediatrics, College of Medicine, University of Iowa, Iowa City, Iowa, USA.

${ }^{3}$ Departments of Biochemistry \& Molecular Biology and Pediatrics, University of Arkansas for Medical Sciences, Little Rock, Arkansas, USA.

${ }^{4}$ To whom correspondence should be addressed. (e-mail: veng@uiowa.edu)
}

Previous studies of bone marrow histology, iron kinetics, and peripheral blood reticulocyte concentrations are consistent with the observed postnatal decrease in $\mathrm{Hb}$ production (5-7). This decrease results from increased oxygen availability and resultant downregulation of erythropoietin (Epo) synthesis and release (810). After the first week of life, erythropoiesis continues at a low rate and the rate of $\mathrm{Hb}$ elimination exceeds its production (4).

In anemic VLBW and ELBW preterm infants, a better understanding of postnatal erythropoiesis is crucial in evaluating their ability to compensate for blood loss due to the multiple clinical phlebotomies that those who are critically ill require. Knowledge of their postnatal erythropoiesis rate would also help in evaluating the potential for enhancing erythropoiesis using strategies such as Epo administration with a goal of substantially reducing or eliminating RBC transfusions.

Previous studies of neonatal erythropoiesis assumed specific structural pharmacodynamic (PD) models to describe the regulation of postnatal $\mathrm{Hb}$ production rate over time $(11,12)$. In these models, the postnatal $\mathrm{Hb}$ production was assumed to be stimulated by Epo through a stimulation function. The stimulation function was related to plasma Epo concentrations by an $E_{\max }$ model $(11,12)$. While useful, this approach is highly dependent on defining the correct relationship between the plasma Epo levels and the $\mathrm{Hb}$ stimulation rate. 
In this work, we introduce a new method that utilizes simple mass balance principles to calculate the amount of $\mathrm{Hb}$ produced after birth and subsequently utilize nonparametric cubic spline functions to estimate the postnatal $\mathrm{Hb}$ production rate. This novel approach does not assume any structural PD model to describe the $\mathrm{Hb}$ simulation rate or its functional relationship to the plasma Epo concentration. This results in a more robust and rational approach for evaluating postnatal erythropoiesis in newborn infants when compared to earlier methods.

The two specific objectives of the present study are (1) to present a novel $\mathrm{Hb}$ mass balance-based semiparametric method that utilizes infant $\mathrm{Hb}$ data from the first 30 postnatal days ("month of life" hereafter) to evaluate the dynamic changes in postnatal erythropoiesis rate in newborn infants, and (2) to apply this method to $\mathrm{Hb}$ data from 79 critically ill VLBW and ELBW preterm anemic infants to estimate the cumulative amount of $\mathrm{Hb}$ produced, to study the changes in neonatal erythropoiesis rate during the first month of life, and to determine the inter-subject variability in postnatal $\mathrm{Hb}$ production.

\section{MATERIALS AND METHODS}

\section{Subjects}

Seventy-nine low birth weight preterm anemic infants (including 27 infants from our previous study (12)), less than 29 weeks GA being cared for in the Neonatal Intensive Care Unit (NICU) at the University of Iowa Children's Hospital, were enrolled in this study. The study was approved by the University of Iowa Human Subject Internal Review Board. For each subject, at least one parent or legal guardian provided written informed consent. Inclusion criteria included treatment with expectation of survival and respiratory distress requiring mechanical ventilation. Exclusion criteria included hematological diseases (other than anemia associated with phlebotomy loss and prematurity), diffuse intravascular coagulation, thrombosis, and transfusion requirements that were emergent and did not allow controlled sampling.

Clinically ordered laboratory phlebotomy blood samples from birth through the end of the first 30 postnatal days were weighed and recorded immediately after collection. The weight of the blood collection tube was subtracted from the total weight of tube and blood sample, and this blood sample weight was converted to the volume of blood removed based on the estimated specific gravity of whole blood of 1.05 (13). The $\mathrm{Hb}$ mass removed with each phlebotomy was calculated by multiplying the volume of blood removed by the $\mathrm{Hb}$ concentration measured at that time. The decision to treat with RBC transfusions was made by the physician in accordance with NICU guidelines (14). For all transfusions, the volume of packed RBCs administered (85\% Hct) was $15 \mathrm{~mL} / \mathrm{kg}$.

\section{Mass Balance-Based Approach}

The total $\mathrm{Hb}$ present at any time $t$ after birth, $H b_{\mathrm{T}}(t)$, can be calculated as:

$$
H b_{\mathrm{T}}(t)=H b_{\mathrm{B}}(t)+H b_{\mathrm{p}}(t)+H b_{\mathrm{TR}}(t)
$$

where $H b_{\mathrm{T}}(t)$ is the total amount of hemoglobin present in circulation at any time $t ; H b_{\mathrm{B}}(t)$ is the amount of hemoglobin present at time of birth $(t=0)$ that are still present at time $t$; $H b_{\mathrm{P}}(t)$ is the amount of hemoglobin produced after birth that are still present in circulation at time $t ; H b_{\mathrm{TR}}(t)$ is the amount of hemoglobin transfused after birth that are still present in circulation at time $t$. The disposition of $\mathrm{Hb}$ was assumed to be lifespan based (i.e., based on removal of RBCs from the circulation through cellular aging/senescence) (15).

\section{Hb present at Time of Birth that are still Present at Time $t$ $\left(H b_{\mathrm{B}}(t)\right)$}

Disposition of $\mathrm{Hb}$ Present at Birth in the Absence of Phlebotomies The $\mathrm{Hb}$ present at the time of birth comes from RBCs produced in utero up until the time of birth $(t=0)$. These RBCs are produced during a period of rapid fetal growth and stimulated erythropoiesis under hypoxic intrauterine conditions. In addition, RBC lifespan varies with gestational age (GA) at birth (i.e., the time between last menstrual period and day of delivery of an infant) and is less than that in healthy adults $(16,17)$. The mathematical model that accounts for both the non-steady state (non-SS) in utero $\mathrm{RBC}$ production and changing fetal RBC lifespan over time has been detailed in our previous work (18). In brief, the in utero body weight, $B W(G A)$, which increases rapidly over time, can be expressed as:

$B W(G A)=\left\{\begin{array}{cc}A \cdot G A^{4}+B \cdot G A^{3}+C \cdot G A^{2}+D \cdot G A+E & G A>154 \\ M \cdot\left(e^{\gamma \cdot G A}-1\right) & 0<G A \leq 154\end{array}\right.$

where $G A$ is the gestational age at birth of the infant measured in days and $A, B, C, D, E, M$, and $\gamma$ are the fixed parameters that were set equal to previously reported values (Table I) (18). The in utero erythropoiesis rate, $R(t)$, is considered to be proportional to the in utero body weight and is expressed as:

$R(t)=k \cdot B W(t+G A) \quad t \leq 0$

where $B W(t)$ is the in utero body weight at time $t, k$ is the scaling/proportionality factor that relates the in utero growth to fetal erythropoiesis rate, and $t$ is the time relative to birth $(t=0)$. To account for in utero changes in RBC lifespan with advancing GA, the fetal RBC lifespan, $L(t)$, was assumed to vary linearly with time and can be expressed as:

$L(t)=L(0)+a t \quad t \leq 0$

where $\alpha$ is the slope parameter describing the rate of change in fetal RBC lifespan with time and $L(0)$ represents the RBC lifespan at the time of birth $(t=0)$.

The final model (derivation detailed in our previous work (18)) used in calculating the amount of $\mathrm{Hb}$ present in RBCs produced in utero and remaining in circulation after birth at time $t$ can be given as: 
Table I. Parameter Estimates Obtained by Fitting Eq. 2 to birth Weight-ga data Extracted from Arbuckle et al. (31) and Previously Reported by kuruvilla et al. (18).

\begin{tabular}{lllll}
\hline & Female, Singleton & Male, Singleton & Male, Twin & \multicolumn{1}{c}{ Female, Twin } \\
\hline$A\left(\mathrm{~g} / \mathrm{day}^{-4}\right)$ & $-1.60 \times 10^{-5}$ & $-1.21 \times 10^{-5}$ & $-9.09 \times 10^{-6}$ & $-1.28 \times 10^{-5}$ \\
$B\left(\mathrm{~g} / \mathrm{day}^{-3}\right)$ & $1.28 \times 10^{-2}$ & $9.37 \times 10^{-3}$ & $6.72 \times 10^{-3}$ & $1.01 \times 10^{-2}$ \\
$C\left(\mathrm{~g} / \mathrm{day}^{-2}\right)$ & -3.66 & -2.53 & -1.73 & -2.87 \\
$D\left(\mathrm{~g} / \mathrm{day}^{-1}\right)$ & $4.61 \times 10^{2}$ & $3.0 \times 10^{2}$ & $1.98 \times 10^{2}$ & $3.67 \times 10^{2}$ \\
$E(\mathrm{~g})$ & $-2.15 \times 10^{4}$ & $-1.30 \times 10^{4}$ & $-8.40 \times 10^{3}$ & $-1.78 \times 10^{4}$ \\
$M(\mathrm{~g})$ & 30.70 & 46.83 & 25.65 & 13.61 \\
$\gamma\left(\mathrm{day}^{-1}\right)$ & $1.86 \times 10^{-2}$ & $1.65 \times 10^{-2}$ & $1.97 \times 10^{-2}$ & $2.27 \times 10^{-2}$ \\
\hline
\end{tabular}

$H b_{\mathrm{B}}=t\left\{\begin{array}{cc}M C H \cdot k\left[\frac{M}{\gamma} \cdot S_{1}(t)+M \cdot S_{2}(t)+S_{3}\right. \\ M C H \cdot k \cdot S_{4}(t) & t \leq(p-G A)(1+\alpha)+L(0) \\ L(0) \geq t>(p-G A)(1+\alpha)+L(0)\end{array}\right.$

where $p=154$ days (Eq. 2) and

$S_{1}(t)=e^{\gamma \cdot p}-e^{\gamma}\left(G A+\frac{t-L(0)}{1+\alpha}\right) \quad$ (6) $\quad S_{2}(t)=G A-p+\frac{t-L(0)}{1+\alpha}$

$S_{3}=\left\{\begin{array}{cc}\frac{A}{5} \cdot\left(G A^{5}-p^{5}\right)+\frac{B}{4} \cdot & \left(G A^{4}-p^{4}\right)+\frac{C}{3} \cdot\left(G A^{3}-p 3\right)+\frac{D}{2} \cdot\left(G A^{2}-p^{2}\right)+E \cdot(G A-p) \quad G A \geq p \\ 0 & G<p\end{array}\right.$

$$
\begin{gathered}
S_{4}(t)=\frac{A}{5}\left(G A^{5}-\left(G A+\frac{t-L(0)}{1+\alpha}\right)^{5}\right)+\frac{B}{4} \cdot\left(G A^{4}-\left(G A+\frac{t-L(0)}{1+\alpha}\right)^{4}\right)+\frac{C}{3} \\
\cdot\left(G A^{3}-\left(G A+\frac{t-L(0)}{1+\alpha}\right)^{3}\right)+\frac{D}{2} \cdot\left(G A^{2}-\left(G A+\frac{t-L(0)}{1+\alpha}\right)^{2}\right)+E \\
\cdot\left(\frac{L(0)-t}{1+\alpha}\right)
\end{gathered}
$$

where $\mathrm{MCH}$ is the mean corpuscular $\mathrm{Hb}$ for the $\mathrm{RBCs}$ present at birth and was set as equal to $37.5 \mathrm{pg} / \mathrm{cell}(11,12)$.

Disposition of $\mathrm{Hb}$ Present at Birth in the Presence of Phlebotomies Newborn VLBW and ELBW preterm infants are subjected to substantial number of phlebotomies for clinical testing purposes; accordingly, Eq. 5 must be corrected to accurately account for the perturbations in $\mathrm{Hb}$ levels caused by the phlebotomies. The loss of fetal RBCs from infant's circulation was accounted for by introducing a phlebotomy correction factor as previously described $(11,12,18)$. Details of the phlebotomy correction are described in the Appendix.

\section{Hb Transfused after Birth that are still Present at Time $t$ $\left(H b_{\mathrm{TR}}(t)\right)$}

Disposition of Transfused $\mathrm{Hb}$ in the Absence of Phlebotomies $\mathrm{The} \mathrm{Hb}$ from multiple $\mathrm{RBC}$ transfusions $\left(H b_{\mathrm{TR}}\right)$ were accounted for through superposition by adding the $\mathrm{Hb}$ mass transfused with transfusion and then accounting for the linear rate of decline of the transfused RBCs. This linear rate of decline arises from assuming normal hematologic steady-state conditions and a constant RBC life span in the adult RBC donor subjects (19). Thus, the behavior of the transfused RBCs is given by:

$H b_{\mathrm{TR}}(t)=\sum_{j=1}^{N T R} H b_{\mathrm{TRj}}(t) \quad H b_{\mathrm{TR}}(0)=0$ 
where $N T R$ is the number of RBC transfusions and $H b_{\mathrm{TRj}}(t)$ represents the hemoglobin amount remaining at time $t$ from the $j^{\text {th }}$ transfusion and is given by:

$H b_{\mathrm{TRj}}(t)\left\{\begin{array}{cc}\frac{F_{\mathrm{T}} \cdot H b_{\mathrm{TR}}\left(t_{j}\right) \cdot\left(L_{\mathrm{TR}_{\mathrm{j}}}+t_{j}-t\right)}{L_{\mathrm{TRj}}} & t_{j} \leq t \leq t_{j}+L_{\mathrm{TRj}}(11) \\ 0 & \text { otherwise }\end{array}\right.$

where $t_{j}$ is the time of the $j^{\text {th }}$ transfusion, $L_{\mathrm{TRj}}$ is the lifespan of transfused RBCs from the $j^{\text {th }}$ transfusion, and $F_{\mathrm{T}}$ is the fraction of transfused RBCs surviving immediately after the transfusion and was set equal to $0.875(11,12)$.

Disposition of Transfused $\mathrm{Hb}$ in the Presence of Phlebotomies The fraction of transfused $\mathrm{Hb}$ remaining after each phlebotomy and the phlebotomy correction factor are calculated and applied as described earlier. Although all RBC transfusions were administered over a 3- to 4-h time period, the effect of the transfusion on the $\mathrm{Hb}$ mass was approximated assuming that the cells were administered as a bolus.

\section{Hb Produced after Birth that are still Present at Time t $\left(H b_{\mathbf{P}}(t)\right)$}

The hemoglobin produced after birth, $H b_{\mathrm{P}}(t)$, can then be calculated as:

$H b_{\mathrm{p}}(t)=H b_{\mathrm{T}}(t)-H b_{\mathrm{B}}(t)-H b_{\mathrm{TR}}$

where the $H b_{\mathrm{T}}(t)$ is the total hemoglobin amount present in circulation at time $t$, while the $H b_{\mathrm{B}}(t)$ and $H b_{\mathrm{TR}}(t)$ are calculated as described earlier.

In the absence of phlebotomies, Eq. 11 can be used to calculate the absolute amount of $\mathrm{Hb}$ produced after birth. But in the presence of multiple phlebotomies, Eq. 11 can only be used to calculate the absolute amount of $\mathrm{Hb}$ produced after birth that is remaining after phlebotomies. Hence, the net amount of $\mathrm{Hb}$ produced after birth (including the amount removed by phlebotomies) would be greater than that calculated from Eq. 11. To account for the $\mathrm{Hb}$ removed due to phlebotomies, Eq. 11 can be modified by incorporating the phlebotomy correction factor (Eq. 16):

$H b_{\mathrm{p}}(t)=\frac{H b_{\mathrm{T}}(t)-H b_{\mathrm{B}}(t)-H b_{\mathrm{TR}}(t)}{\text { Phlebotomy Correction Factor }}$

Equation 12 can be used to calculate the total amount of $\mathrm{Hb}$ produced from birth to the end of the study period. In this study, we assumed that the RBC lifespan of the produced RBCs was longer than the 30-day study period (i.e., none of the RBCs produced after birth is removed from the circulation during the study period). Equation 13 can be then be used to calculate the cumulative amount of $\mathrm{Hb}$ produced after birth throughout the study period.

\section{Iman Conover Regression Fit to $H b_{\mathrm{P}}(t)$}

The Iman Conover regression is a well-established nonparametric method that makes use of the rank transform approach in regression (20). This method is particularly advantageous when the dependent variable is a monotonic function of the independent variable, even when the monotonic relationship is nonlinear in nature (20). Since the cumulative $\mathrm{Hb}$ produced after birth is monotonically increasing with time, the Iman Conover regression fit to the estimated Hb-time data (Eq. 12) was used for predicting the cumulative amount of $\mathrm{Hb}$ produced after birth over time.

\section{Nelder-Mead Objective Function Minimization and Cubic Spline Fit}

The cumulative amount of $\mathrm{Hb}$ produced after birth (Eq. 12) is dependent on several parameters used to describe $H b_{\mathrm{B}}(t)$ and $H b_{\mathrm{TR}}(t)$. As described earlier, two separate models were used to describe the disposition of $H b_{\mathrm{B}}(t)$ and $H b_{\mathrm{TR}}(t)$. The disposition of $\mathrm{Hb}$ produced before birth that remains in circulation at any time $t$ after birth (Eqs. 5-9), $H b_{\mathrm{B}}(t)$, is dependent on the following model parameters: $L(0), k$, and $\alpha$. Similarly, for the transfused $\mathrm{Hb}$ (Eqs. 10-11), the disposition of the $\mathrm{Hb}$ administered for each transfusion, $H b_{\mathrm{TR}}(t)$, is dependent on the lifespan of the transfused RBCs, $L_{\mathrm{TRj}}$. These parameters were optimized by minimizing the sum of the absolute value of the residuals as defined by the objective function:

Objective Function $=\sum_{i=1}^{N}\left|H b_{\mathrm{p}}\left(t_{i}\right)-\widehat{H b}_{\mathrm{p}}\left(t_{i}\right)\right|$

where $H b_{\mathrm{P}}\left(t_{i}\right)$ is calculated as described in Eq. 13, and $\hat{H} b_{\mathrm{P}}\left(t_{i}\right)$ is the predicted amount of $\mathrm{Hb}$ produced at time $t_{i}$ from the regression fit to the data. The value of the objective function (Eq. 14) was minimized by the Nelder-Mead simplex method (21).

The final predicted cumulative amount of $\mathrm{Hb}$ produced was then represented by a nonparametric cubic smoothing spline function (22). The $\mathrm{Hb}$ production rate (i.e., neonatal erythropoiesis rate) at any time during the first month of life was then evaluated as the first derivative of this cubic spline. Finally, the body weight-scaled $\mathrm{Hb}$ production rate was calculated during the first month of life by normalizing the estimated $\mathrm{Hb}$ production rate by the body weight at that time.

\section{Data Analysis}

Data analyses were performed in $\mathrm{R}$ version 3.0.3 using the RStudio integrated development environment $(23,24)$. The Nelder-Mead objective function minimization, Iman Conover nonparametric regression, and cubic spline fits were all conducted using WINFUNFIT, a Windows (Microsoft) version evolved from the general nonlinear regression program FUNFIT (25). A regression slope $t$ test $($ slope $\neq 0$ ) was used to evaluate the linear correlation observed between infant gestational age and the mean body weight-scaled $\mathrm{Hb}$ production rate over the first week and month of life. Statistical differences were considered to be significant for values of $P<0.05$.

The total blood volume was assumed to be proportional to the infant body mass. The total $\mathrm{Hb}$ amount present in infant circulation at any time $t, H b_{\mathrm{T}}(t)$, was estimated by multiplying the measured $\mathrm{Hb}$ concentration times the total blood volume at time $t$. 


\section{RESULTS}

\section{Subject Characteristics}

The mean GA of the 79 newborn subjects was 25.6 weeks (range, 22.4 to 28.6 weeks). Thirty-five males (27 singletons and 8 twins) and 44 females (35 singletons and 9 twins) were studied. The infants underwent an average of 104 phlebotomies (range, 36 to 215). The mean birth weight was $0.768 \mathrm{~kg}$ (range, 0.412 to $1.487 \mathrm{~kg}$ ). The average number of RBC transfusions administered during the study period was 3.7 (range, 0 to 10 ).

\section{Mass Balance-Based Semiparametric Approach}

Figure 1 shows an overlay of $H b_{\mathrm{T}}(t)$, i.e., the total amount of $\mathrm{Hb}$ present in infant circulation at any time $t$ after birth, and $H b_{\mathrm{TR}}(t)+H b_{\mathrm{B}}(t)$, i.e., the sum of the transfused $\mathrm{Hb}$ (Eqs. 10-11) and the $\mathrm{Hb}$ produced in utero up to time of birth remaining in circulation at any time $t$ after birth (Eqs. 5-9) for a representative infant study subject. The sum of $H b_{\mathrm{B}}(t)$ and $H b_{\mathrm{TR}}(t)$ was corrected to account for the loss of $\mathrm{Hb}$ due to multiple phlebotomies. The difference between the model predicted solid line and the individual data points provides an estimate of the cumulative amount of $\mathrm{Hb}$ produced by the infant during the first month of life, before accounting for the loss of $\mathrm{Hb}$ due to phlebotomies.

The Iman Conover regression fit to the calculated cumulative amount of $\mathrm{Hb}$ produced after accounting for the loss due to phlebotomies (Eq. 13) is shown in Fig. 2a. Figure 2b shows the nonparametric cubic smoothing spline fit to $\hat{H} b_{\mathrm{P}}(t)$, the predicted cumulative amount of $\mathrm{Hb}$ produced at time $t$ from the Iman Conover regression fit to the data. The mean $( \pm \mathrm{SD})$ parameter estimates obtained from the Nelder-Mead minimization of the

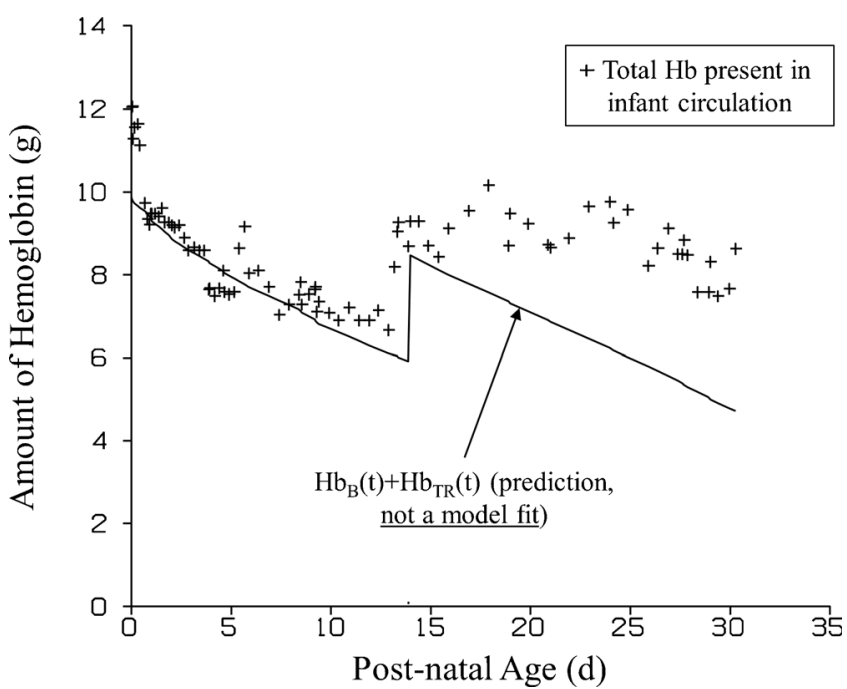

Fig. 1. $\mathrm{Hb}$ amount present in the circulation of a representative infant during the first month of life. The individual data shown $(+)$ represent $H b_{T}(t)$, the total amount of $\mathrm{Hb}$ present in infant circulation at any time during the first month of life (Eq.1). The solid line represents $H b_{B}(t)+H b_{T R}(t)$, the sum of transfused $\mathrm{Hb}$ and the $\mathrm{Hb}$ produced in utero prior to birth and remaining in the infant's circulation following birth after accounting for phlebotomy loss. The solid line represents a prediction and is not a model fit. The difference between the solid line and the data points $(+)$ represent $H b_{P}(t)$, the amount of $\mathrm{Hb}$ produced by the infant at that time before accounting for phlebotomy loss objective function (Eq. 14) were $L(0)=35.5 \pm 12.8$ days, $k=$ $1.70 \times 10^{7} \pm 0.65 \times 10^{7} \mathrm{RBCs} / \mathrm{day} / \mathrm{g}, \alpha=0.83 \pm$ 0.59 , and $L_{T R}=51.6 \pm 26.3$ days.

The cumulative $\mathrm{Hb}$ produced during the first month of life and the dynamic changes in body weight-scaled $\mathrm{Hb}$ production rate during the same period are displayed for four representative subjects in Fig. 3. The results of the PD analysis utilizing the mass balance-based semiparametric method for the 79 low birth weight infants are summarized in Table II. Finally, to test the influence of $\mathrm{GA}$ at birth on the postnatal $\mathrm{Hb}$ production, the mean body weight-scaled $\mathrm{Hb}$ production rate over the first week of life for all study subjects was plotted against their GA (Fig. 4). A significant positive correlation was found between GA and the mean body weight-scaled $\mathrm{Hb}$ production rate over the first week of life $(P<0.05)$. A similar significant positive correlation was also found between $\mathrm{GA}$ and the mean body weight-scaled $\mathrm{Hb}$ production rate over the first month of infant life $(P<0.05)$.

\section{DISCUSSION}

In premature newborns, the relationship between Epo and $\mathrm{Hb}$ levels is complex. In addition to the impact of Epo on stimulating $\mathrm{Hb}$ production, there are several other factors including $\mathrm{P}_{50}, 2$, 3-diphosphoglycerate, cardiac output, and mixed venous oxygen that also play a role in the Epo response (26-28). Attempts to develop a structured model to describe neonatal erythropoiesis should factor all these variables into the model to successfully evaluate the postnatal $\mathrm{Hb}$ production in these infants. Previous attempts to evaluate the PD of Epo focused solely on structured parametric PD models that related to $\mathrm{Hb}$ production to the plasma Epo concentrations $(11,12,15,29,30)$. Due to the inherent complexities associated with evaluating neonatal erythropoiesis, a nonparametric or semiparametric approach would be more suitable for this purpose.

This study introduces a mass balance-based semiparametric approach to evaluate the PD of Epo in newborn infants. The advantage of this approach over previously developed methods for describing neonatal erythropoiesis is that this approach does not a priori assume any specific structured PD model for describing postnatal $\mathrm{Hb}$ production. Instead, the cumulative amount of $\mathrm{Hb}$ produced over the first month of life is calculated by mass balance principles, and nonparametric methods (cubic spline) are utilized in evaluating the dynamic changes in $\mathrm{Hb}$ production rate over the first month of life.

As illustrated in Figs. 1 and 2, the mass balance-based method permits successful estimation of the cumulative amount of $\mathrm{Hb}$ produced over the first month of life. The estimated $\mathrm{Hb}$ data were fitted with the Iman Conover nonparametric regression to determine the cumulative amount of $\mathrm{Hb}$ produced postnatally at any time during the first month of life (Fig. 2a). The Iman Conover regression ensures that cumulative amount of $\mathrm{Hb}$ produced is monotonically increasing with time. This is important because, by definition, the cumulative amount $\mathrm{Hb}$ produced cannot decrease with time. Furthermore, since the first derivative of the cumulative amount of $\mathrm{Hb}$ produced yields the postnatal $\mathrm{Hb}$ production rate, a hypothetical decrease in the cumulative amount of $\mathrm{Hb}$ produced would indicate a negative postnatal $\mathrm{Hb}$ production rate in the infant. This would be physiologically meaningless.

The predicted cumulative amount of $\mathrm{Hb}$ produced as obtained from the Iman Conover regression was fitted with a nonparametric cubic smoothing spline (Fig. 2b). In contrast to 

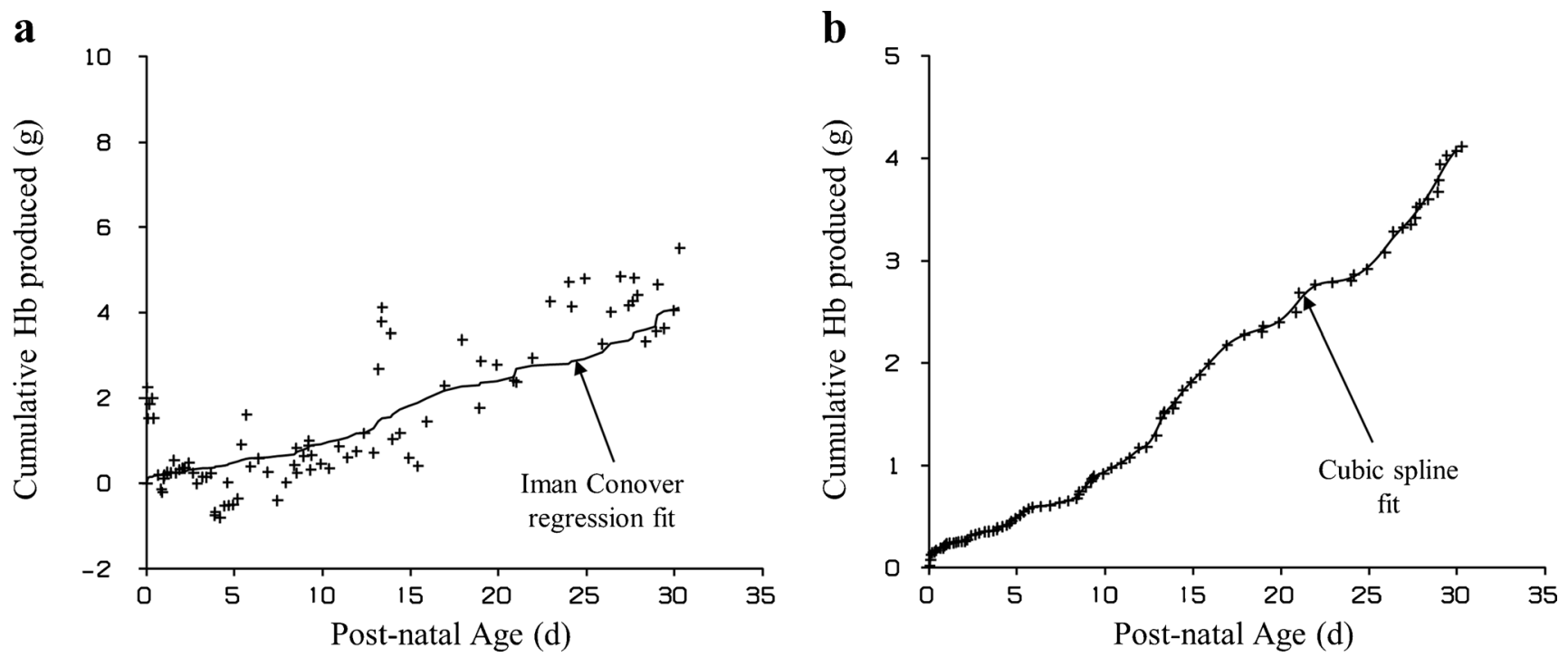

Fig. 2. Iman Conover nonparametric regression fit and subsequent cubic spline fit to infant $\mathrm{Hb}$ data. The amount of $\mathrm{Hb}$ produced during the first month of life $\left(H b_{P}(t)\right)$ was estimated using Eq. 12. a These data were then fit with the Iman Conover nonparametric regression. The solid line represents the regression fit to the calculated $H b_{P}(t)$ data $(+)$. b The final predicted cumulative $\mathrm{Hb}$ amounts $(+)$ were then fit with a nonparametric cubic smoothing spline function (solid line)

a

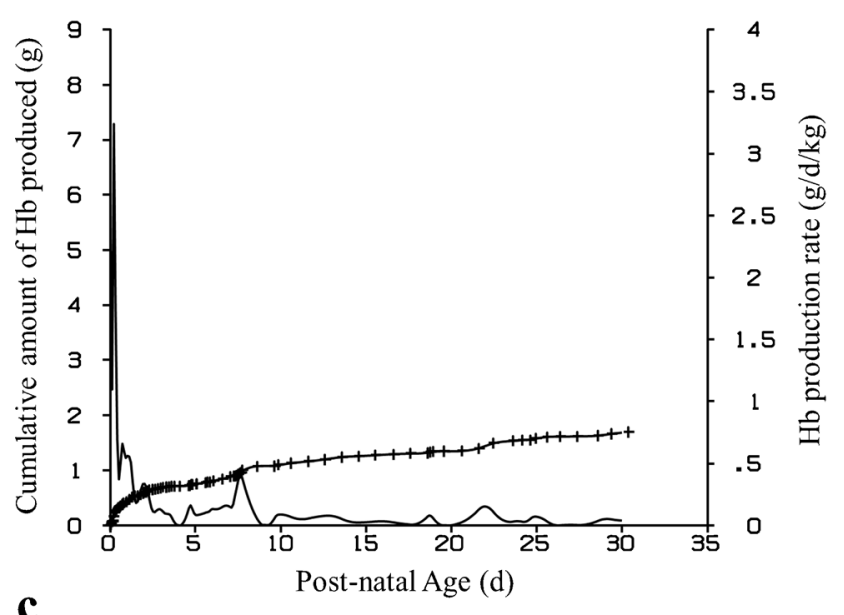

c

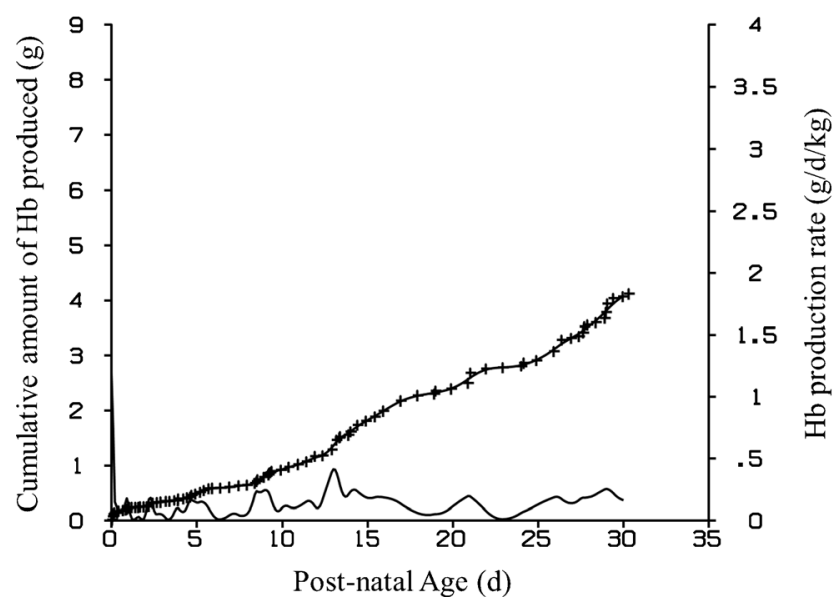

b
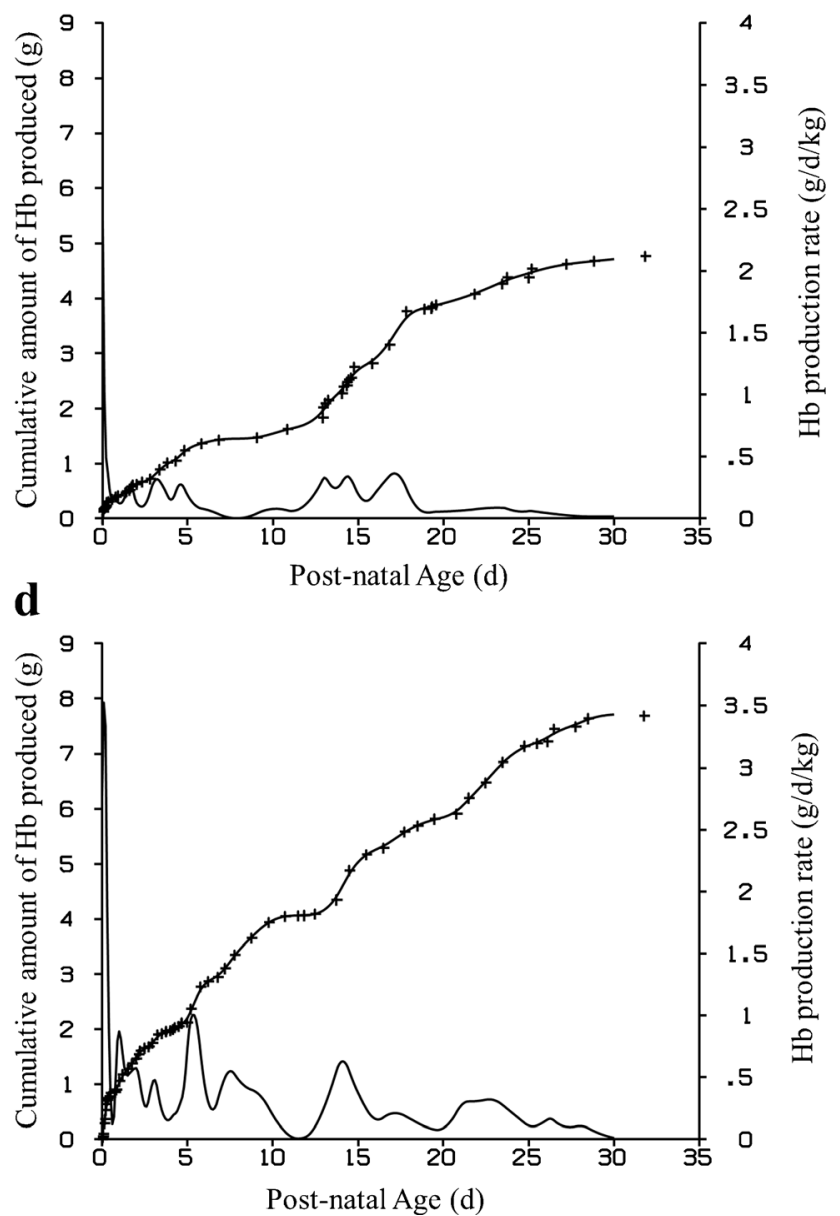

Fig. 3. a-d Dynamic change in postnatal Hb production during the first month of life for four representative subjects. The solid line represents the cubic smoothing spline fit to the estimated cumulative $\mathrm{Hb}$ produced $(+)$. The other solid line represents the dynamic changes in body weight-scaled $\mathrm{Hb}$ production rate during the first month of life 
Table II. Summary of Infant Pharmacodynamic Estimates from the Hb mass Balance-based Semiparametric method ( $n=79)$

\begin{tabular}{lllll}
\hline & $\begin{array}{l}\text { Cumulative amount of } \mathrm{Hb} \\
\text { produced during the first week } \\
\text { of life }(\mathrm{g})\end{array}$ & $\begin{array}{l}\text { Cumulative amount of Hb } \\
\text { produced during the first month } \\
\text { of life }(\mathrm{g})\end{array}$ & $\begin{array}{l}\text { Mean Hb production rate } \\
\text { during the first week of life } \\
(\mathrm{g} / \mathrm{day} / \mathrm{kg})\end{array}$ & $\begin{array}{l}\text { Mean Hb production rate } \\
\text { during the first month of life } \\
(\mathrm{g} / \mathrm{day} / \mathrm{kg})\end{array}$ \\
\hline Mean & 1.50 & 6.60 & 0.24 & 0.23 \\
SD & 0.72 & 3.44 & 0.12 & 0.12 \\
\hline
\end{tabular}

$S D$ standard deviation

lower order polynomial functions, cubic smoothing splines have the advantage of being more flexible, and thus are better able to describe the local behavior of a curve. When studying neonatal erythropoiesis, especially in anemic VLBW and ELBW preterm infants, the rate of postnatal $\mathrm{Hb}$ production can vary considerably based on several factors including allogeneic RBC transfusions, endogenous Epo levels, and disease conditions. These dynamic changes in erythropoiesis are best captured by fitting the flexible cubic smoothing spline function as displayed in Fig. 2b.

Using this approach, we were able to successfully estimate the postnatal $\mathrm{Hb}$ production rate in the newborn infants (Fig. 3). We observed that the postnatal $\mathrm{Hb}$ production rate was high at birth and then dropped to lower levels within the first few days of life. After the first week, the $\mathrm{Hb}$ production rate remained at lower levels until the end of the 1-month study period. This drop in postnatal $\mathrm{Hb}$ production after birth is in agreement with previous reports and is consistent with the observed increase in oxygen availability and resultant downregulation of Epo synthesis and release (8-10).

The estimated mean cumulative amount of $\mathrm{Hb}$ produced for the 79 infants (mean body weight, $0.768 \mathrm{~kg}$ ) over the first month of life was $6.6 \mathrm{~g}$ (SD, 3.4) (Table II) and is similar to previously reported value of $4.7 \mathrm{~g}(\mathrm{SD}, 3.3)$ in our previously reported study of 14 preterm infants (11). The mean estimated body weightscaled $\mathrm{Hb}$ production rate over the first month of life was $0.226 \mathrm{~g} /$ day/kg (SD, 0.119) and was below our previously reported maximum postnatal $\mathrm{Hb}$ production rate of $0.43 \mathrm{~g} / \mathrm{day} / \mathrm{kg}^{3 / 4}(12)$.

Finally, the importance of GA of the newborn infant on the postnatal $\mathrm{Hb}$ production was investigated (Fig. 4). It was observed that the mean body weight-scaled $\mathrm{Hb}$ production rate during the first week of life increased with GA. This is in agreement with our previous population PD study that identified GA as the most important covariate affecting the PD of endogenous Epo in anemic VLBW preterm infants (12).

\section{Limitations of the study}

The mass balance-based approach detailed in this work is a "semiparametric" approach that does not a priori assume any specific structured model to evaluate the amount of $\mathrm{Hb}$ produced after birth $\left(H b_{\mathrm{P}}(t)\right)$. It does, however, define two separate models to describe the disposition of $\mathrm{Hb}$ from the other two populations of RBCs present in the infant circulation (Eq. 1). These two include the $\mathrm{Hb}$ produced before birth that remain in circulation after birth at time $t$, $H b_{\mathrm{B}}(t)$, and the $\mathrm{Hb}$ transfused to infants at any time during the study that remain in circulation at time $t, H b_{T R}(t)$. The disposition of $\mathrm{Hb}$ from these two RBC populations was modeled as described earlier using Eqs. 5-9 and Eqs. 10-11.

In this study, the measured $\mathrm{Hb}$ data demonstrated moderate variability of about $20 \%$. Since the proposed method is based on mass balance principles, the $\mathrm{Hb}$ data measured in the infants should be as accurate and precise as possible. In future, with improved infant $\mathrm{Hb}$ measurements, this proposed method could be applied to even more accurately quantify postnatal $\mathrm{Hb}$ production in newborn infants. This study also assumes that none of the RBCs produced after birth is removed from circulation. Thus, the effects of neocytolysis, storage of immature RBCs in the spleen, and other factors that may play a small

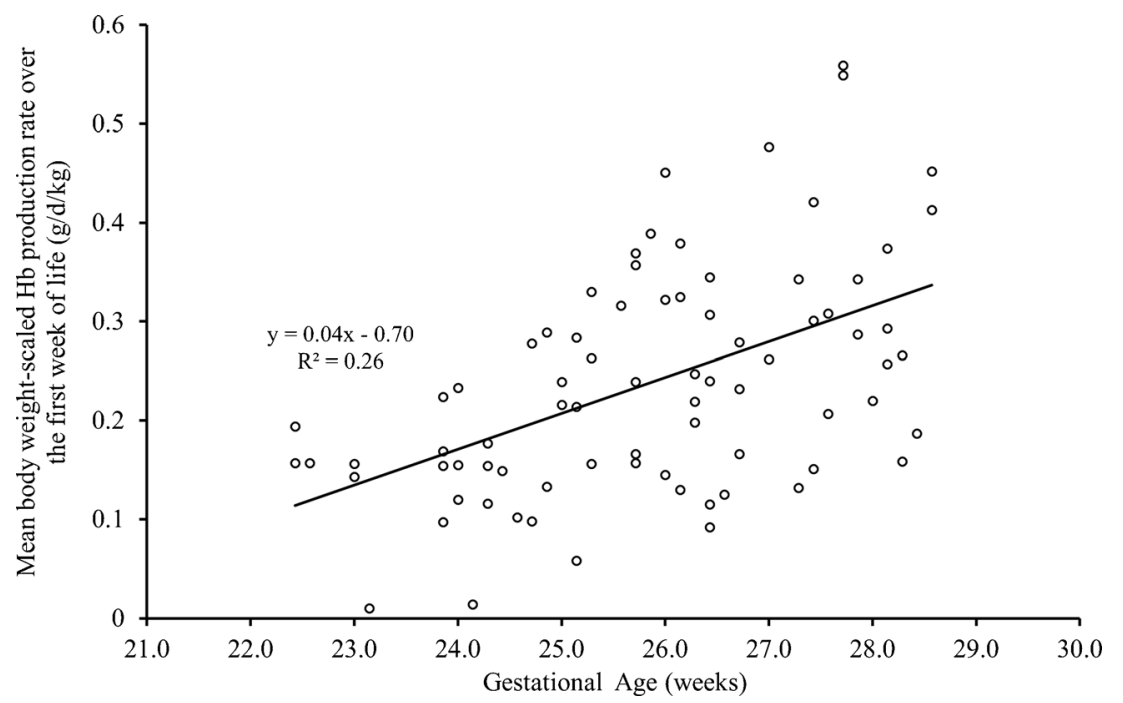

Fig. 4. Influence of $\mathrm{GA}$ on the postnatal $\mathrm{Hb}$ production. The individual data points represent the estimated body weight-scaled postnatal $\mathrm{Hb}$ production rate over the first week of life $v s$. the GA for the 79 VLBW and ELBW anemic preterm study infants 
role in the removal of newly produced RBCs from circulation are not considered in this study.

\section{Clinical Significance}

Compared to healthy adults, erythropoiesis in newborns, especially VLBW and ELBW preterm anemic infants, has not been well studied. It is during the first month of life, when their severity of illness is usually at its peak, that anemic preterm infants experience a large number clinical phlebotomies, require multiple allogeneic RBC transfusions, and exhibit a rapid increase in body weight, all of which affects their ability to produce $\mathrm{Hb}$. In this study, mass balance principles and nonparametric regression techniques were used to evaluate the dynamic changes in postnatal $\mathrm{Hb}$ production during the first month of life. Results from the present study of 79 anemic low birth weight preterm infants indicate that the GA of the infant is an important factor in determining the postnatal erythropoiesis in the infant. Infants with higher GA were able to produce greater amounts of postnatal $\mathrm{Hb}$ normalized for body weight, making the more mature infants less susceptible to developing anemia. Finally, the knowledge gained from this study on the dynamic changes in postnatal $\mathrm{Hb}$ production will be helpful in evaluating the potential treatment strategies for improvement of erythropoiesis, such as Epo therapy, aimed at reducing or eliminating RBC transfusions.

\section{CONCLUSION}

In summary, this work introduces a mass balance-based semiparametric approach that does not assume any structural PD model for describing the dynamic changes associated with neonatal erythropoiesis during the first month of life. Due to the inherent complexities associated with neonatal erythropoiesis in VLBW and ELBW preterm anemic infants, this more flexible approach that is based on fewer assumptions offers a more direct and suitable way for evaluating the PD effect of Epo and other erythropoiesis stimulating agents compared to earlier methods. Future work with this approach includes studying the relationship between the $\mathrm{Hb}$ production rates and the plasma Epo concentrations and identifying covariates that in addition to GA have a significant effect on the postnatal $\mathrm{Hb}$ production in VLBW and ELBW infants.

\section{ACKNOWLEDGEMENTS}

The authors acknowledge helpful discussions with Guohua An, Ph.D. (University of Iowa), regarding methodological and theoretical aspects of the present study. We also express appreciation to Mark A. Hart for editorial and secretarial assistance. Also appreciated are the many outstanding clinical research contributions of Iowa's neonatal research nurse team who including Gretchen Cress, R.N., M.P.H.; Karen Johnson, R.N.; Jin Zhou, R.N.; and Ruthann Schrock, R.N. The gracious help provided by the University of Iowa clinical laboratory staff led by Mitchell J. Owen, MT (ASCP), and Mary Capper, MT (ASCP)SH, and overseen by Matthew D. Krasowski, M.D., Ph.D. was essential in the success of this study. This work would not have been possible without the permission of the parents of study subjects to allow their infants to participate. Finally, we are grateful to the Sysmex Corporation, Kobe, Japan (http:// www.sysmex.co.jp/en/index.html) for the generous loan of the Sysmex XE-2100 automatic hematology analyzer used in this study. This work was supported by National Institutes of Health (NIH) US Public Health Service Program Project Grant 2 P01 HL046925 and the National Center for Research Resources, a part of the NIH, Grant Number UL1TR000039.

Author Contributions D.N., R.L.S., and J.A.W. performed experiments; D.J.K., R.L.S., D.N., and J.A.W. analyzed data. D.J.K. prepared the first draft of the manuscript. P.V-P, D.N., R.L.S., J.A.W., and D.M. provided additional ideas, discussions, and helped in critical review. All authors approved the final version of the manuscript.

\section{Compliance with Ethical Standards}

Disclosures No conflicts of interest, financial or otherwise, are declared by the authors.

\section{GLOSSARY}

\begin{tabular}{|c|c|}
\hline $\mathrm{RBC}$ & Red blood cell \\
\hline $\mathrm{Hb}$ & Hemoglobin \\
\hline VLBW & Very low birth weight infant \\
\hline ELBW & Extremely low birth weight infant \\
\hline GA & Gestational age \\
\hline Epo & Erythropoietin \\
\hline Non-SS & Non-steady state \\
\hline NICU & Neonatal intensive care unit \\
\hline$H b_{\mathrm{T}}(t)$ & $\begin{array}{l}\text { Total amount of hemoglobin present in } \\
\text { circulation at any time } t\end{array}$ \\
\hline$H b_{\mathrm{B}}(t)$ & $\begin{array}{l}\text { Hemoglobin amount present at birth }(t=0) \\
\text { which still are present at time } t\end{array}$ \\
\hline$H b_{\mathrm{P}}(t)$ & $\begin{array}{l}\text { Hemoglobin amount produced after birth } \\
\text { still present in circulation at time } t\end{array}$ \\
\hline$H b_{\mathrm{TR}}(t)$ & $\begin{array}{l}\text { Hemoglobin amount transfused after birth } \\
\text { still present in circulation at time } t\end{array}$ \\
\hline$\hat{H} b_{\mathrm{P}}(t)$ & $\begin{array}{l}\text { Predicted amount of } \mathrm{Hb} \text { produced at } \\
\text { time } t \text { obtained from regression fit to } \mathrm{Hb} \text { data }\end{array}$ \\
\hline $\mathrm{BW}(t)$ & In utero body weight at time $t$ \\
\hline $\mathrm{MCH}$ & Mean corpuscular hemoglobin \\
\hline Hct & Hematocrit \\
\hline$L_{\mathrm{TRj}}$ & $\begin{array}{l}\text { Lifespan of transfused RBCs from } \\
\text { the } j^{\text {th }} \text { transfusion }\end{array}$ \\
\hline$F_{\mathrm{T}}$ & $\begin{array}{l}\text { Fraction of transfused RBCs surviving } \\
\text { immediately after the transfusion }\end{array}$ \\
\hline$R(t)$ & In utero erythropoiesis rate \\
\hline A & Rate of change in in utero fetal RBC lifespan \\
\hline $\mathrm{K}$ & $\begin{array}{l}\text { Scaling factor that relates in utero growth } \\
\text { to fetal erythropoiesis rate }\end{array}$ \\
\hline $\mathrm{L}(0)$ & Lifespan of RBCs present at birth $(t=0)$ \\
\hline NTR & Number of donor RBC transfusions \\
\hline $\mathrm{F}_{\mathrm{RMi}}$ & $\begin{array}{l}\text { Fraction of total } \mathrm{Hb} \text { remaining } \\
\text { after } i^{\text {th }} \text { phlebotomy }\end{array}$ \\
\hline $\mathrm{PD}$ & Pharmacodynamic \\
\hline$T$ & Time \\
\hline
\end{tabular}

\section{APPENDIX}

\section{Correction for phlebotomies}

Let us consider the $i^{\text {th }}$ phlebotomy was performed at time $t_{\mathrm{pi}}$ that removed a certain fraction of $\mathrm{Hb}$ from circulation. The fraction remaining after the $i^{\text {th }}$ phlebotomy, $F_{\mathrm{RMi}}$, is given by Eq. 15 : 
$F_{\mathrm{RMi}}=\frac{H b_{T}\left(t_{\mathrm{pi}}\right)-H b_{\mathrm{RMi}}}{H b_{T}\left(t_{\mathrm{pi}}\right)}$

where $H b_{\mathrm{RMi}}$ is the hemoglobin removed due to the $i^{\text {th }}$ phlebotomy at time $t_{\mathrm{pi}}$. For multiple phlebotomies, the phlebotomy correction factor can be calculated as shown in Eq. 16,

Phlebotomy Correction Factors

$$
=\left\{\begin{array}{cc}
\prod_{i=j}^{q} F_{\mathrm{RMi}} & \text { if } \\
1 & \text { otherwise }
\end{array}\right.
$$

where the fraction remaining after each phlebotomy is ordered from the first to the last phlebotomy, $j$ is the first phlebotomy after entry of the RBCs into the systemic circulation and $q$ is the last phlebotomy prior to the current time $t$. In this case, $j$ represents the first phlebotomy after the RBCs are introduced into the circulation, and thus the Eq. 16 now can be written as Eq. 17. The derived phlebotomy correction factor is then multiplied with Eq. 5 and Eq. 11 to account for loss of RBCs due to multiple clinical phlebotomies.

Phlebotomy Correction Factor

$$
=\left\{\begin{array}{cc}
\prod_{i=1}^{q} F_{\mathrm{RMi}} & \text { if } \\
1 & \text { otherwise }
\end{array}\right.
$$

\section{REFERENCES}

1. Struss RG. Anaemia of prematurity: Pathophysiology and treatment. Blood Rev. 2010;24(6):221-5.

2. Kett JC. Anemia in infancy. Blood. 2012;1599:1678.

3. Dallman PR. Anemia of prematurity. Annu Rev Med. 1981;32(1):143-60.

4. Finne P, Halvorsen S. Regulation of erythropoiesis in the fetus and newborn. Arch Dis Child. 1972;47(255):683.

5. Kalpaktsoglou PK, Emery JL. The effect of birth on the haemopoietic tissue of the human bone marrow. A histological study. Br J Haematol. 1965;11(4):453-60.

6. Garby L, SjÖLin S, Vuille J-C. Studies on erythro-kinetics in infancy. Acta Pædiatrica. 1962;51(2):245-54.

7. Seip M. The reticulocyte level, and the erythrocyte production judged from reticulocyte studies, in newborn infants during the first week of life. Acta Paediatr. 1955;44(4):355-69.

8. Kett JC. Anemia in infancy. Pediatr Rev. 2012;33(4):186-7.

9. Kaplan E, Hsu KS. Determination of erythrocyte survival in newborn infants by means of Cr51-labelled erythrocytes. Pediatrics. 1961;27(3):354-61.
10. Sturgeon P. Volumetric and microscopic pattern of bone marrow in normal infants and children: II Cytologic pattern. Pediatrics. 1951;7(5):642-50.

11. Freise KJ, Widness JA, Veng-Pedersen P. Erythropoietic response to endogenous erythropoietin in premature very low birth weight infants. J Pharmacol Exp Ther. 2010;332(1):229-37.

12. Saleh MI, Nalbant D, Widness JA, Veng-Pedersen P. Population pharmacodynamic analysis of erythropoiesis in preterm infants for determining the anemia treatment potential of erythropoietin. Am J Physiol Regul Integr Comp Physiol. 2013;304(9):R77281.

13. Trudnowski RJ, Rico RC. Specific gravity of blood and plasma at 4 and $37^{\circ}$ C. Clin Chem. 1974;20(5):615-6.

14. Strauss RG. How I, transfuse red blood cells and platelets to infants with the anemia and thrombocytopenia of prematurity. Transfusion (Paris). 2008;48(2):209-17.

15. Krzyzanski W, Ramakrishnan R, Jusko W. Basic pharmacodynamic models for agents that alter production of natural cells. J Pharmacokinet Biopharm. 1999;27(5):467-89.

16. Pearson HA. Life-span of the fetal red blood cell. J Pediatr. 1967;70(2):166-71.

17. Brace RA, Langendörfer C, Song T-B, Mock DM. Red blood cell life span in the ovine fetus. Am J Physiol Regul, Integr Comp Physiol. 2000;279(4):R1196-204.

18. Kuruvilla D, Widness J, Nalbant D, Schmidt R, Mock D, VengPedersen P. A method to evaluate fetal erythropoiesis from postnatal survival of fetal RBCs. AAPS J. 2015;342:1-9.

19. Mock DM, Widness JA, Veng-Pedersen P, Strauss RG, Cancelas JA, Cohen RM, et al. Measurement of posttransfusion red cell survival with the biotin label. Transfus Med Rev. 2014;28(3):11425 .

20. Iman RL, Conover WJ. The use of the rank transform in regression. Technometrics. 1979;21(4):499-509.

21. Nelder JA, Mead R. A simplex method for function minimization. Comput J. 1965;7(4):308-13.

22. Reinsch C. Smoothing by spline functions. Numer Math. 1967;10(3):177-83.

23. R Core Team. R: A language and environment for statistical computing. Vienna, Austria: R Foundation for Statistical Computing; 2014.

24. Racine JS. RStudio: a platform-independent IDE for $\mathrm{R}$ and Sweave. J Appl Econ. 2012;27(1):167-72.

25. Veng-Pedersen P. Curve fitting and modeling in pharmacokinetics and some practical experiences with NONLIN and a new program FUNFIT. J Pharmacokinet Biopharm. 1977;5(5):51331.

26. Doyle JJ. The role of erythropoietin in the anemia of prematurity. Semin Perinatol. 1997;21(1):20-7.

27. Stockman III JA, Garcia JF, Oski FA. The anemia of prematurity: factors governing the erythropoietin response. $\mathrm{N}$ Engl J Med. 1977;296(12):647-50.

28. Hågå $\mathrm{P}$, Meberg $\mathrm{A}$, Halvorsen S. Plasma erythropoietin concentrations during the early anemia of prematurity. Acta Pædiatrica. 1983;72(6):827-31.

29. Krzyzanski W, Wyska E. Pharmacokinetics and pharmacodynamics of erythropoietin receptor in healthy volunteers. NaunynSchmied Arch Pharmacol. 2008;377(4-6):637-45.

30. Woo S, Krzyzanski W, Jusko W. Target-mediated pharmacokinetic and pharmacodynamic model of recombinant human erythropoietin (rHuEPO). J Pharmacokinet Pharmacodyn. 2007;34(6):849-68.

31. Arbuckle TE, Wilkins R, Sherman GJ. Birth weight percentiles by gestational age in Canada. Obstet Gynecol. 1993;81(1):39-48. 